\title{
Surface Grafting of Polyesters onto Carbon Nanofibers and Electric Properties of Conductive Composites Prepared from Polyester-grafted Carbon Nanofibers
}

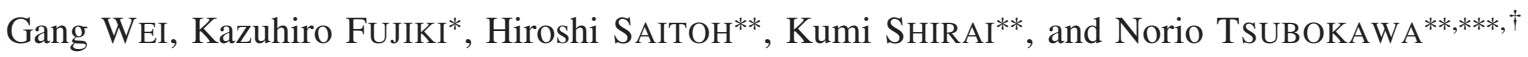 \\ Graduate School of Science and Technology, Niigata University, 8050 Ikarashi 2-no-cho, Niigata 950-2181, Japan \\ ${ }^{*}$ Division of Life and Health Sciences, Joetsu University of Education, 1 Yamayashiki, Joetsu 943-8521, Japan \\ ${ }^{* *}$ Department of Material Science and Technology, Faculty of Engineering, Niigata University, \\ 8050 Ikarashi 2-no-cho, Niigata 950-2181, Japan \\ *** Center for Transdisciplinary Research, Niigata University, 8050 Ikarashi 2-no-cho, Niigata 950-2181, Japan
}

(Received October 10, 2003; Accepted January 27, 2004)

\begin{abstract}
The grafting of polyesters onto carbon naonfiber, such as vapor grown carbon fiber (VGCF) and high density VGCF (HDVGCF), by the anionic ring-opening alternating copolymerization of epoxides with cyclic acid anhydrides initiated by potassium carboxylate (COOK) groups previously introduced onto these surfaces was investigated. The introduction of COOK groups onto these carbon nanofiber surfaces was successfully achieved by the neutralization of carboxyl groups with $\mathrm{KOH}$. Carboxyl groups were introduced onto these surfaces by oxidation of these nanofibers with nitric acid and mixture of nitric acid with sulfuric acid. It was found that the anionic ring-opening alternating copolymerization of epoxides with cyclic acid anhydrides was successfully initiated by COOK groups on the surface to give the corresponding polyester-grafted carbon nanofiber. The dispersibility of carbon nanofiber in THF was remarkably improved by the grafting of polyesters onto the surface. The electric resistance of composite prepared from the polyester-grafted carbon nanofiber and poly(ethylene glycol) suddenly increased in methanol vapor over $10^{3}$ times, and returned to initial resistance when it was transferred into dry air. These results indicate that the composite can be used as a novel gas sensor.

KEY WORDS Carbon Nanofiber / Surface Grafting / Polyester / Anionic Ring-opening Copolymerization / Conductive Composite / Gas Sensor /
\end{abstract}

Vapor grown carbon fiber (VGCF) is a new class of carbon nanofiber differentiated from carbon nanotube in its production method and its lower cost. It is also different from the conventional pitch-based and polyacrylonitrile-based carbon fiber in its nano-sized diameter. ${ }^{1}$ VGCF is known to be in the form of annular carbon layers arranged like a tree ring structure. The carbon rods have a high aspect ratio and a nano-sized diameter ranging from 50 to $200 \mathrm{~nm}$, which is between those of conventional carbon fibers $(5-10 \mu \mathrm{m})$ and of carbon nanotubes $(1-50 \mathrm{~nm}){ }^{2}$ Due to their unique physical properties, such as excellent thermal and electrical conductivity, good mechanical behavior and lower cost of production, VGCF has attracted considerable attention in the last ten years. ${ }^{3,4}$

VGCF is usually used as a reinforcing agent, an adsorbent and a conductive filler. Recently, they are also used in batteries for electrode and separator. ${ }^{5,6}$ In most cases, they are used in the form of composite where the polymer is needed as matrices. However, due to the very low surface free energy, nano-size and low bulk density, it is difficult to disperse VGCF in polymer matrices uniformly.

We expect that the grafting of polymer onto VGCF surface is preferable for the modification of the surface. In the composite prepared from polymer-grafted VGCF and polymer, the VGCF is uniformly dispersed in polymer matrix. It provides the best possible adhesion and allows for optimal transfer of stress from the matrix to the fiber.

It is well known that the oxygen containing groups are successfully introduced onto carbon fiber surface by the treatments with oxidative agents, such as nitric acid, ${ }^{7}$ plasma, ${ }^{8}$ corona, ${ }^{9}$ air, ${ }^{10}$ and carbon dioxide ${ }^{11}$ and by electrochemical treatments. ${ }^{12}$ These functional groups can be used for the grafting sites of various polymers.

We have reported the radical grafting of polymers onto carbon materials, such as carbon black and carbon fiber surface, initiated by various initiating groups previously introduced onto the surface. ${ }^{13-16}$ More recently, we have succeeded in the grafting by ligandexchange reaction of ferrocene containing polymers with aromatic rings of carbon fiber ${ }^{17,18}$ and by direct $\gamma$-ray irradiation of polymer-adsorbed carbon black and VGCF. ${ }^{19,20}$

In this paper, we investigated the grafting of polyesters onto VGCF surface by the anionic ring-opening

${ }^{\dagger}$ To whom correspondence should be addressed (E-mail: ntsuboka@eng.niigata-u.ac.jp). 

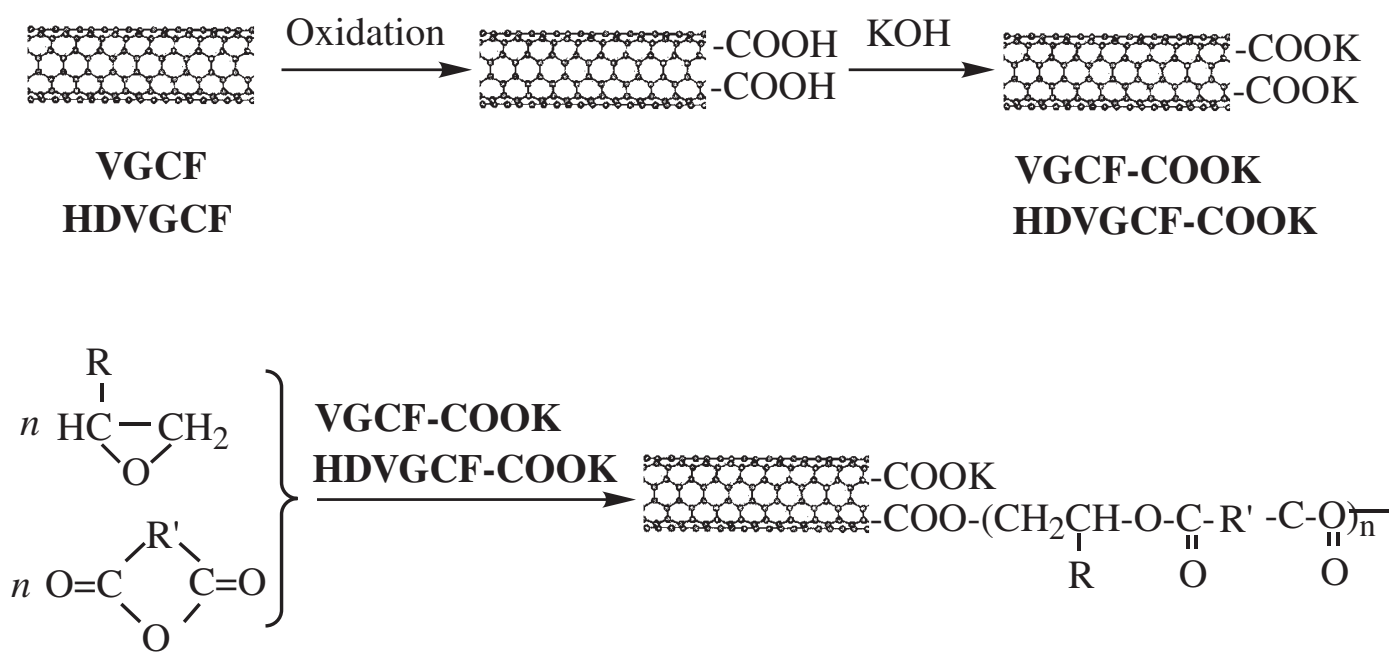

Scheme 1.

alternating copolymerization of epoxides with cyclic acid anhydrides initiated by COOK groups introduced onto the VGCF surface (Scheme 1). In addition, the electric properties of conductive composite prepared from polyester-grafted VGCF were investigated.

\section{EXPERIMENTAL}

\section{Material and Reagents}

Vapor growth carbon fiber (VGCF) and high-density vapor growth carbon fiber (HDVGCF) were supplied from Showa Denko K.K. Japan. The VGCF has about 8 graphite winding layers with $150 \mathrm{~nm}$ diameter and $10-15 \mu \mathrm{m}$ length. The specific surface area of VGCF is $13 \mathrm{~m}^{2} / \mathrm{g}$. The bulk density of VGCF and HDVGCF are $0.04 \mathrm{~g} / \mathrm{cm}^{3}$ and $0.095 \mathrm{~g} / \mathrm{cm}^{3}$, respectively.

Maleic acid anhydride (MAn), phthalic acid anhydride (PAn), styrene oxide (SO), and epichlorohydrin $(\mathrm{ECH})$ were obtained from Kanto Chemical Co., Inc., Japan. MAn and PAn were purified by recrystalization from benzene followed by the sublimation under reduced pressure. $\mathrm{ECH}$ and $\mathrm{SO}$ were dried over $\mathrm{CaH}_{2}$ for $12 \mathrm{~h}$, then distilled under reduced pressure.

Poly ( $\varepsilon$-caprolactone) (PCL; $\left.M_{n}=2000\right)$ and poly(ethylene glycol) (PEG; $M_{n}=3400$ ) were obtained from Aldrich Chemical Co., Inc. and used without further purification.

\section{Introduction of COOK Groups onto VGCF Surface}

The introduction of COOK groups onto VGCF (HDVGCF) surface was achieved by the neutralization of carboxyl groups with $\mathrm{KOH}$. The oxidation of VGCF to introduce carboxyl groups onto the surface was achieved by two methods. One was oxidation with concentrated nitric acid (35\% and 60\%) for $5 \mathrm{~h}$ at $110^{\circ} \mathrm{C}$. The other was oxidation with the mixture of concentrated sulfuric acid (96-98\%) and nitric acid
(97\%) $\left(\mathrm{H}_{2} \mathrm{SO}_{4} / \mathrm{HNO}_{3}=4 / 1(\mathrm{v} / \mathrm{v})\right)$ at $70{ }^{\circ} \mathrm{C}$ for $3 \mathrm{~h}$ after dispersing with ultrasonic wave $(150 \mathrm{~W})$ irradiation for $5 \mathrm{~min}$ (No cutting of the fiber was observed under the condition).

After the oxidation, the resulting VGCF was filtrated and washed with pure water until the filtrate turned to neutral. Then, it was treated at $1050{ }^{\circ} \mathrm{C}$ for about $15 \mathrm{~s}$. The content of carboxyl groups introduced onto the surface was determined by the titration. ${ }^{21}$

The introduction of COOK groups onto VGCF was achieved as follows. Into a flask, $2.0 \mathrm{~g} \mathrm{VGCF}-\mathrm{COOH}$, $100 \mathrm{~mL} 10 \% \mathrm{KOH}$ aqueous solution were charged and the reaction mixture was refluxed with stirring at $70{ }^{\circ} \mathrm{C}$ for $6 \mathrm{~h}$. After reaction, the resultant mixture was filtrated, and washed with pure water until filtrate turned to neutral, then dried in vacuum at $110^{\circ} \mathrm{C}$.

Anionic Ring-opening Alternating Copolymerization of Epoxides with Cyclic Acid Anhydrides Initiated by COOK Groups Introduced onto the Surface of $V G C F$

Into a flask, $0.10 \mathrm{~g}$ VGCF-COOK, $0.01 \mathrm{~mol}$ of epoxides (SO or ECH) and cyclic acid anhydride (PAn or MAn) and $0.02 \mathrm{~g}$ of 18 -crown-6 were charged. The flask was cooled with a liquid nitrogen bath, thawed three times, and sealed under high vacuum. The polymerization was carried out at $120^{\circ} \mathrm{C}$ under stirring with a magnetic stirrer. After the reaction, the reaction mixture was poured into a large excess of methanol to precipitate the polyester-grafted VGCF and ungrafted polyester, and the precipitate was dried in vacuum.

\section{Percentage of Grafting}

To remove the ungrafted polyester, the product was dispersed in THF and the dispersion was allowed to centrifuge at $1.0 \times 10^{4} \mathrm{rpm}$. The VGCF precipitated was dispersed again in THF and centrifuged. The pro- 
Table I. Carboxyl group content of unterated and oxidized carbon nanofibers

\begin{tabular}{lcccc}
\hline & \multicolumn{4}{c}{ Carboxyl group $(\mathrm{mmol} / \mathrm{g})$} \\
\cline { 2 - 5 } Nanofiber & \multirow{2}{*}{ Unterated } & \multicolumn{3}{c}{ Oxidized } \\
\cline { 3 - 5 } & & $35 \% \mathrm{HNO}_{3}$ & $60 \% \mathrm{HNO}_{3}$ & $\mathrm{HNO}_{3} / \mathrm{H}_{2} \mathrm{SO}_{4}$ \\
\hline VGCF & 0.38 & 2.2 & 3.5 & 4.8 \\
HDVGCF & 0.31 & - & - & 1.8 \\
\hline
\end{tabular}

cedures were repeated until no more polyester could be detected in the supernatant solution. The percentage of grafting was determined by the following equation:

$$
\text { Grafting }(\%)=A / B \times 100
$$

where $A$ is the polyester grafted $(\mathrm{g})$ and $B$ is VGCF used $(\mathrm{g})$. The amount of polyester grafted onto the VGCF surface was determined by weight loss when the polyester-grafted VGCF was heated from room temperature to $500^{\circ} \mathrm{C}$ under nitrogen at $10^{\circ} \mathrm{C} / \mathrm{min}$ with a thermal analyzer (Thermogravimetric Analyzer TGA-50 by Shimadzu Corp.).

\section{Dispersibility of Polyester-grafted VGCF in Solvent}

Polyester-grafted VGCF $(0.5 \mathrm{~g})$ was dispersed in $100 \mathrm{~mL}$ of solvent and allowed to stand at room temperature.

\section{Measurement of Electric Properties of Composite from Polyester-grafted VGCF}

The conductive composite paste was prepared from polyester-grafted VGCF and PCL (or PEG) using THF as solvent. The polyester-grafted VGCF composite paste was coated onto a comb-like electrode, which was prepared by the screen-printing of conductive $\mathrm{Ag} / \mathrm{Pd}$ paste onto a ceramic plate. The thickness of the composite on the electrode was about $100 \mu \mathrm{m}$. The DC electric resistance of the electrode was recorded by an ohmmeter at $25^{\circ} \mathrm{C}$ every $10 \mathrm{~s}$ in dry air and then in saturated solvent vapor at $25^{\circ} \mathrm{C}$. The details of the preparation of the sensor material and comb-like electrode, the measurement of electric resistance and the response analysis were described in our previous papers. ${ }^{22-26}$

\section{RESULTS AND DISCUSSION}

\section{Introduction of Carboxyl Groups onto VGCF and HDVGCF Surface}

The introduction of COOK groups onto VGCF surface was achieved by the neutralization of carboxyl groups with $\mathrm{KOH}$ aqueous solution. The introduction of carboxyl groups was achieved by two kind of oxidation reaction: one is oxidation of VGCF with the nitric acid (35\% and 60\%) and the other is oxidation with the mixture of concentrated sulfuric acid and ni- tric acid $(4 / 1, v / v)$. The content of $\mathrm{COOH}$ groups after the oxidation is shown in Table I.

As shown in Table $\mathrm{I}, \mathrm{COOH}$ group content of VGCF remarkably increased about 10 times with increasing concentration of nitric acid. In the case of oxidation of VGCF and HDVGCF with the mixture of nitric acid and sulfuric acid, $\mathrm{COOH}$ group content on the surfaces also increased and reached to $4.8 \mathrm{mmol} / \mathrm{g}$ and $1.8 \mathrm{mmol} / \mathrm{g}$, respectively. Carboxyl groups introduced onto HDVGCF was less than those onto VGCF were. This suggests that the structure of VGCF is more easily oxidized than HDVGCF.

It is considered that carboxyl groups are introduced onto not only the edges of VGCF but also wall of VGCF surface. However, based on the reactivity of VGCF, the amount of carboxyl groups introduced onto wall seems to be considerably small.

Carbon nanofibers having carboxyl groups were abbreviated as VGCF-COOH and HDVGCF-COOH. Then, VGCF-COOH and $\mathrm{HDVGCF}-\mathrm{COOH}$ were treated with $\mathrm{KOH}$ aqueous solution and the surface $\mathrm{COOH}$ groups were converted to $\mathrm{COOK}$ groups.

Figure 1 shows the stability of dispersion of (A) untreated HDVGCF, (B) HDVGCF-COOH, and (C) HDVGCF-COOK in water. As shown in Figure 1, untreated HDVGCF precipitated within $1 \mathrm{~d}$, but HDVGCF-COOH and HDVGCF-COOK gave a stable dispersion in water for over 2 weeks. This indicates that HDVGCF surface turned extremely hydrophilic by the introduction of $\mathrm{COOH}$ and $\mathrm{COOK}$ groups.

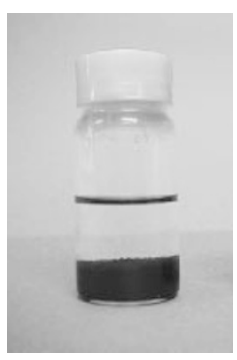

(A)

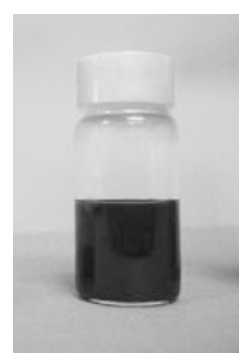

(B)

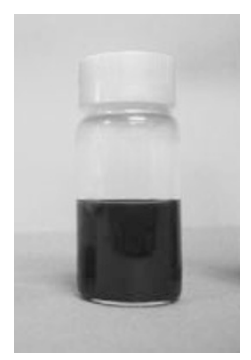

(C)
Figure 1. Dispersibility of (A) untreated HDVGCF, (B) HDVGCF-COOH, and (C) HDVGCF-COOK in water at room temperature. 
Table II. Anionic ring-opening alternating copolymerization of SO with PAn initiated by COOK groups on VGCF

\begin{tabular}{lcccc}
\hline VGCF & $\begin{array}{c}\text { SO } \\
(\mathrm{mol})\end{array}$ & $\begin{array}{c}\text { PAn } \\
(\mathrm{mol})\end{array}$ & $\begin{array}{c}\text { Conversion } \\
(\%)\end{array}$ & $\begin{array}{c}\text { Grafting } \\
(\%)\end{array}$ \\
\hline Untreated & 0.01 & 0.01 & 0 & - \\
VGCF-COOH & 0.01 & 0.01 & 0 & 0 \\
VGCF-COOK & 0.01 & - & 0 & 0 \\
VGCF-COOK & - & 0.01 & 0 & 0 \\
VGCF-COOK & 0.01 & 0.01 & 9.2 & 4.8 \\
\hline
\end{tabular}

VGCF, $0.10 \mathrm{~g} ; 18$-croown- $6,0.02 \mathrm{~g} ; 120^{\circ} \mathrm{C} ; 8 \mathrm{~h}$.

Anionic Ring-opening Alternating Copolymerization $S O$ with PAn Initiated by $\mathrm{VGCF}-\mathrm{COOK}$

We have reported that the anionic ring-opening alternating copolymerization of epoxides with cyclic acid anhydrides are initiated by COOK groups on carbon black surface to give the corresponding polyestergrafted carbon black. ${ }^{27-29}$ Therefore, the ability of VGCF-COOK to initiate the anionic ring-opening alternating copolymerization of SO with PAn was investigated. The results are shown in Table II. The polymerization was carried out in the presence of crown ether to accelerate the polymerization. ${ }^{29}$

As shown in Table II, no polymerization of $\mathrm{SO}$ with PAn was initiated in the presence of untreated VGCF and VGCF-COOH. VGCF-COOK has no ability to initiate the polymerization of SO and PAn alone. On the contrary, the anionic ring-opening alternating copolymerization of SO with PAn was successfully initiated by VGCF-COOK to give the corresponding polyester-grafted VGCF.

Figure 2 shows IR spectra of (A) poly(SO-alt-PAn),

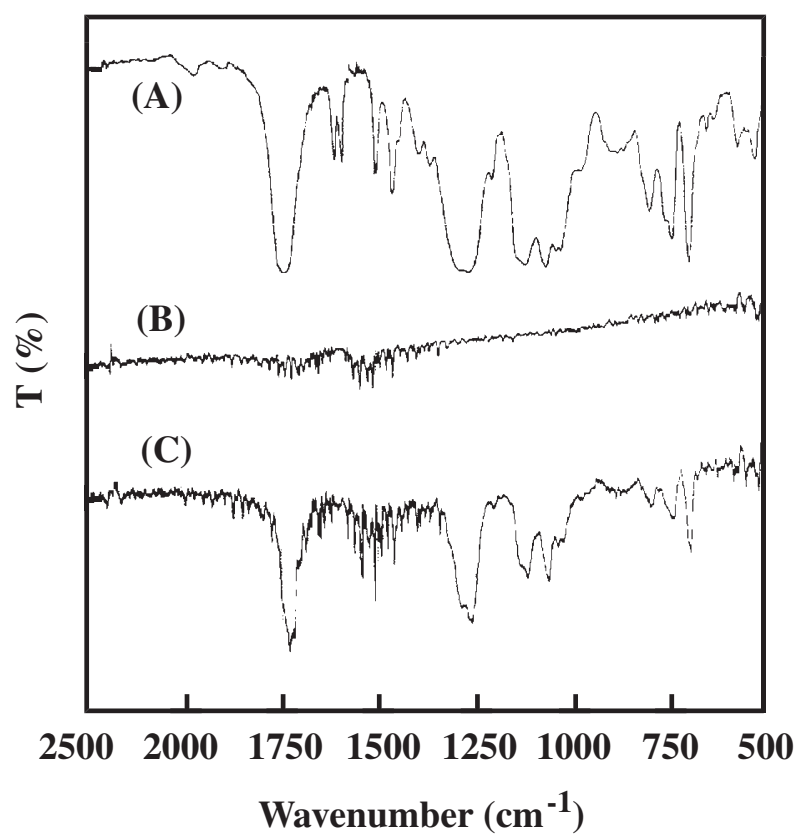

Figure 2. Infrared spectra of (A) poly(SO-alt-PAn), (B) untreated VGCF, and (C) poly(SOU-alt-PAn)-grafted VGCF (grafting $=10.3 \%$ ).

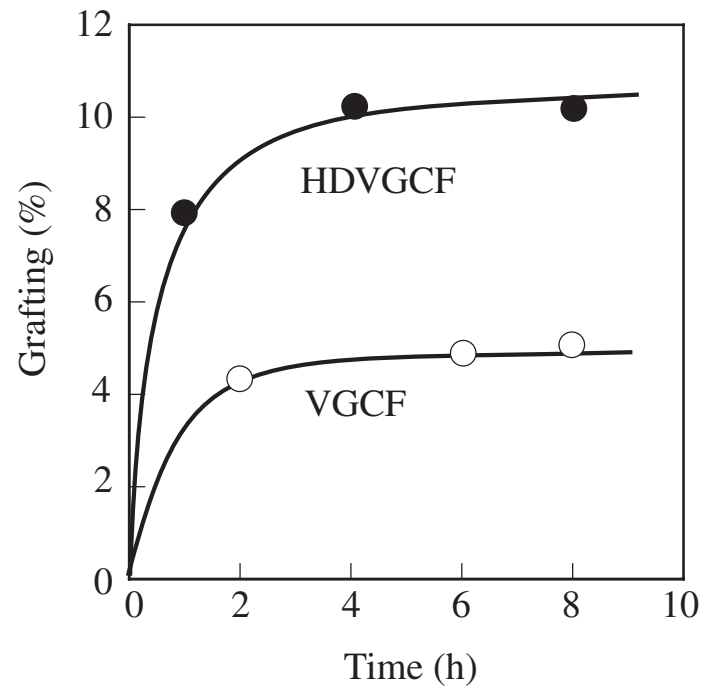

Figure 3. Grafting of poly(SO-alt-PAn) onto HDVGCF and VGCF by the anionic ring-opening alternating copolymerization of SO with PAn initiated by COOK groups on the surface. VGCF, $0.10 \mathrm{~g} ; \mathrm{SO}=\mathrm{PAn}=0.01 \mathrm{~mol} ; 18$-crown- $6,0.02 \mathrm{~g} ; 120^{\circ} \mathrm{C}$.

(B) ungrafted VGCF, and (C) poly(SO-alt-PAn)-grafted VGCF. As shown in Figure 2, IR spectra of poly(SO-alt-PAn)-grafted VGCF show adsorptions characteristic of poly(SO-alt-PAn). These results also show the grafting of poly(SO-alt-PAn) onto VGCF surface.

Grafting of Poly(SO-alt-PAn) onto VGCF and HDVGCF surface

Figure 3 shows time-poly(SO-alt-PAn) grafting curves of the anionic ring-opening alternating copolymerization of SO with PAn initiated by VGCFCOOK and HDVGCF-COOK. It is interesting to note that the percentage of poly(SO-alt-PAn) grafting onto HDVGCF surface is much higher than that onto VGCF surface, regardless of lower COOK group content of HDVGCF.

This may be due to the difference in dispersibility between VGCF-COOK and HDVGCF-COOK in the reaction mixture. HDVGCF-COOK shows good dispersibility in reaction mixture and COOK groups of HDVGCF-COOK were effectively used as initiating sites.

\section{Grafting of Several Polyesters onto HDVGCF Surface}

The anionic ring-opening alternating copolymerization of several epoxides with cyclic acid anhydrides was investigated. Epoxides used were SO and $\mathrm{ECH}$, and cyclic acid anhydride used were PAn and MAn. The results are shown in Figure 4. It was found that HDVGCF-COOK has an ability to initiate the anionic ring-opening alternating copolymerization of epoxides with cyclic acid anhydrides to give the corresponding polyester-grafted HDVGCF. 


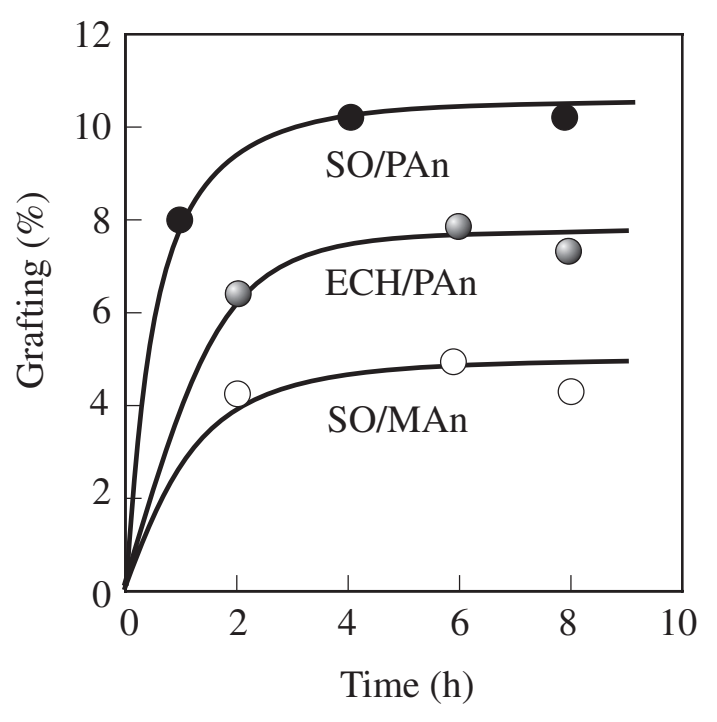

Figure 4. Grafting of polyesters onto HDVGCF by the anionic ring-opening alternating copolymerization of epoxides with cyclic acid anhydrides initiated by COOK groups on the surface. HDVGCF, $0.10 \mathrm{~g}$; epoxide $=$ cyclic acid anhydride $=0.01 \mathrm{~mol}$; 18-crown-6, $0.02 \mathrm{~g} ; 120^{\circ} \mathrm{C}$.
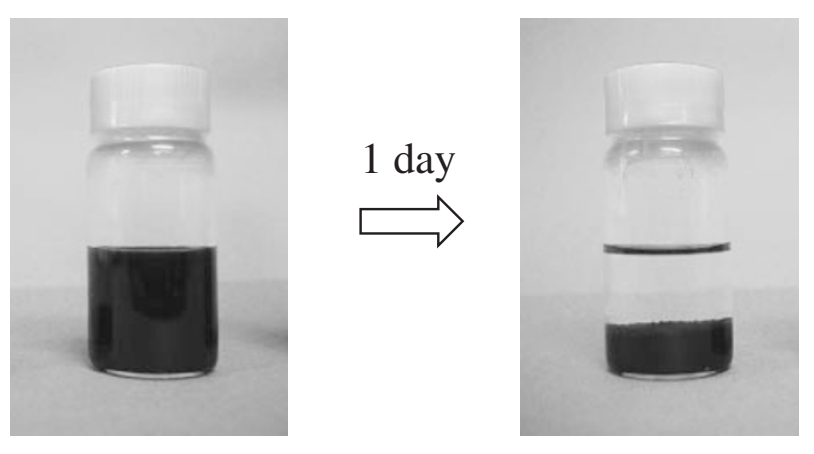

Untreated

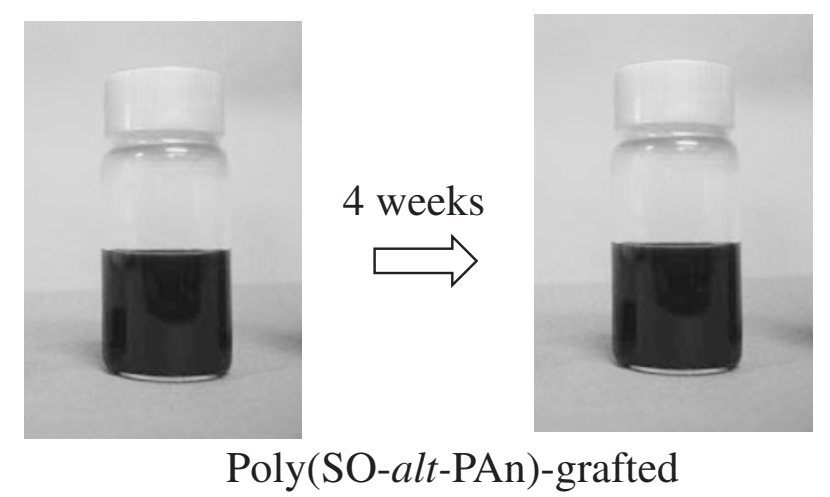

Figure 5. Dispersibility of poly(SO-alt-PAn)-grafted (grafting $=10.3 \%$ ) and ungrafted HDVGCF in THF at room temperature.

Dispersibility of Polyester-grafted HDVGCF in Organic Solvent

Figure 5 shows the stability of dispersion of poly(SO-alt-PAn)-grafted HDVGCF in THF. Untreated HDVGCF precipitated within $1 \mathrm{~d}$ as mentioned above. On the contrary, poly(SO-alt-PAn)-grafted HDVGCF gave a stable dispersion in good solvent for grafted polyester, such as THF. The precipitation of poly(SO-alt-PAn)-grafted HDVGCF was scarcely observed even after 4 weeks.

The grafted polyester chains are mainly grafted onto the edges of VGCF, because of the presence of carboxyl groups on the edges. However, it seems that the grafting of polyester onto wall of VGCF also proceeded. Both polyester chains on edged and wall of VGCF play an important role for the destruction the entanglement.

Electric Properties of Composite from Polyestergrafted $H D V G C F$

Polyester-grafted HDVGCF readily and uniformly dispersed in polyesters and polyethers, such as PCL and PEG, and gave a conductive composite. We have reported that composites prepared from crystalline polymer-grafted carbon black and crystalline polymer can be used as novel gas sensors. ${ }^{22-26}$

Figure 6 shows the relationship between poly(SOalt-PAn)-grafted VGCF content and electric resistance of the poly(SO-alt-PAn)-grafted VGCF/PEG composite. Generally, the resistance decreased very sharply after reaching the percolation threshold, which strongly depended on the filler.

In the case of low poly(SO-alt-PAn)-grafted VGCF content, electric resistance is very high, indicating no conducting network for electric current, and VGCF fibers are isolated in the composite. With increasing VGCF content to a critical VGCF weight fraction (percolation threshold), the VGCF network is formed in the composite, which leads to drastic decrease in electric resistance.

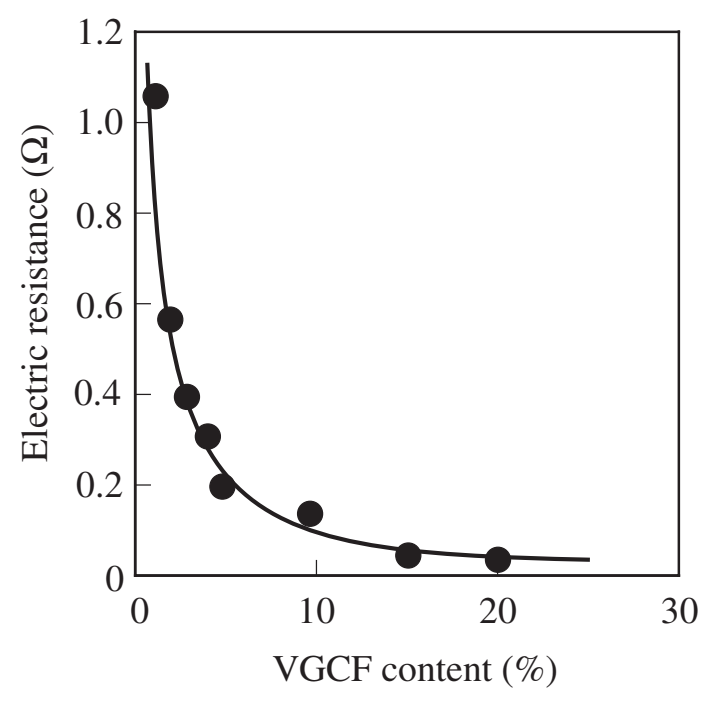

Figure 6. Effect of poly(SO-alt-PAn)-grafted HDVGCF (grafting $=10.3 \%$ ) content on the electric resistance of composite prepared from poly(SO-alt-PAn)-grafted HDVGCF and PEG. 


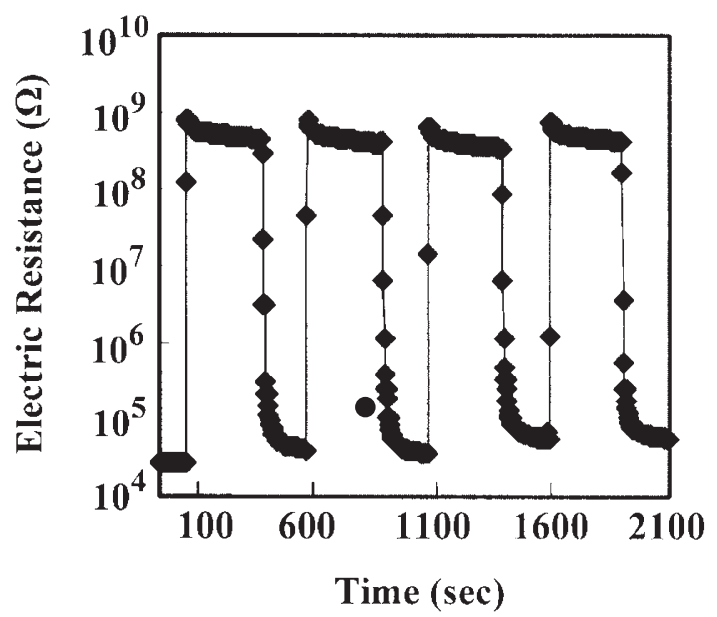

Figure 7. Responsibility of electric resistance of composite prepared from poly(SO-alt-PAn)-grafted HDVGCF (grafting = $10.3 \%$ ) and PCL to acetone vapor at $25^{\circ} \mathrm{C}$. HDVGCF:PCL $=$ $1: 10$.

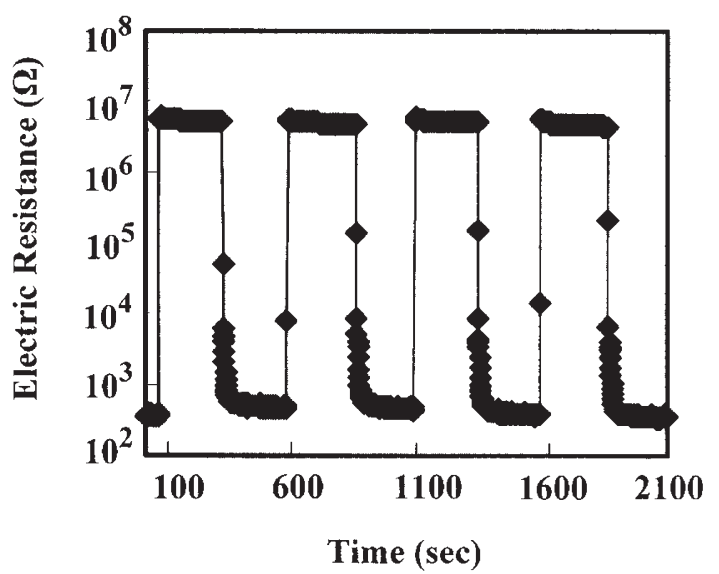

Figure 8. Responsibility of electric resistance of composite prepared from poly(SO-alt-PAn)-grafted HDVGCF (grafting = $10.3 \%$ ) and PEG to methanol vapor at $25^{\circ} \mathrm{C}$. HDVGCF:PEG $=$ $1: 10$.

Electric Properties of Composite from Polyestergrafted HDVGCF in Solvent Vapor

Figure 7 shows the electric resistance change of the composites prepared from poly(SO-alt-PAn)-grafted HDVGCF and PCL in acetone vapor at $25^{\circ} \mathrm{C}$. On the other hand, Figure 8 shows the electric resistance change of the composites prepared from poly(SO-altPAn)-grafted HDVGCF and PEG in methanol vapor at $25^{\circ} \mathrm{C}$. The electric resistance of these composites suddenly increased in acetone or methanol vapor, and returns immediately to initial resistance when the composites were transferred in dry air. The responsiveness of electric resistance was reproducible even after 30 cycles of exposure to methanol vapor and dry air.

This is due to a slight change of gaps between HDVGCF based on the absorption of vapor of a good solvent by grafted chain and matrix polymer. These results suggest the possibility of detection of a slight change of crystalline structure of polymer by the absorption of solvent as a large change of electric resistance of the composite.

\section{CONCLUSIONS}

1. The anionic ring-opening alternating copolymerization of epoxides with cyclic acid anhydride was successfully initiated by COOK groups previously introduced onto VGCF and HDVGCF and the corresponding polyesters were grafted onto the surface.

2. The dispersibility of VGCF and HDVGCF in THF were remarkably improved by the grafting of polyesters onto the surface.

3. The electric resistance of composite prepared from polyester-grafted HDVGCF and PCL or PEG suddenly increased in solvent vapor and returned to initial resistance when it was transferred into dry air.

Acknowledgment. This study was partly supported by a Grant in Aid for Scientific Research from the Ministry of Education, Culture, Sports, Science and Technology of Japan (No. 15360357), which is gratefully acknowledged.

\section{REFERENCES}

1. G. G. Tibbetts, G. L. Doll, D. W. Gorkiewicz, J. J. Moleski, T. A. Perry, C. J. Dasch, and M. J. Balogh, Carbon, 31, 1039 (1993).

2. M. Endo, K. Takeuchi, T. Furuta, T, Kasai, X. Sun, C. Kiang, and M. Dresselhaus, J. Phys. Chem. Solids, 58, 1702 (1997).

3. L. Piraux, B. Nysten, A. Haquenne, J. Issi, M. S. Dresselhaus, and M. Endo, Solid State Commun., 50, 697 (1984).

4. J. Issi and B. Nysten, "Carbon Fibers," J. Donnet, T. Wong, J. Peng, and S. Rebouillat, Ed., Marcel Dekker, New York, N.Y., 1998, pp. 371-462.

5. H. Abe, T. Murai, and K. Zaghib, J. Power Sources, 77, 110 (1999).

6. M. Endo, Y. Kim, T. Hayashi, K. Nishimura, T. Matusita, K. Miyashita, and M. Dresselhaus, Carbon, 39, 1287 (2001).

7. S. Gardner, C. Singamsetty, G. Booth, G. He, and C. Pittman, Carbon, 33, 587 (1995).

8. H. Bubert, X. Ai, S. Haiber, M. Heintze, V. Brüser, E. Pasch, W. Brandl, and G. Marginean, Spectrochim. Acta, Part B: At. Spectrosc., 57, 1601 (2002).

9. E. Desimoni, A. M. Salvi, F. Langerame, and J. F. Watts, J. Electron Spectrosc. Relat. Phenom., 85, 179 (1997).

10. R. Pogue, J. Ye, A. Klosterman, A. Glass, and R. Chartoff, Composites, Part A, 29, 1273 (1998).

11. P. Serp, J. Figueiredo, P. Bertrand, and J. Issi, Carbon, 36, 1791 (1998). 


\section{G. WEI et al.}

12. S. Waseem, S. Gardner, G. Hea, W. Jiang, and C. Pittman, J. Mater. Sci., 33, 3151 (1998).

13. N. Tsubokawa, Prog. Polym. Sci., 17, 417 (1992).

14. N. Tsubokawa and T. Yoshihara, J. Polym. Sci., Part A: Polym. Chem., 31, 2459 (1993).

15. K. Fujiki, N. Motoji, and A. Yoshida, Compos. Interfaces, $\mathbf{3}$, 371 (1996).

16. N. Tsubokawa, Bull. Chem. Soc. Jpn., 75, 2115 (2002).

17. N. Tsubokawa, N. Abe, and Y. Seida, Chem. Lett., 2000, 900.

18. N. Tsubokawa, N. Abe, G. Wei, J. Chen, and S. Saitoh, J. Polym. Sci., Part A: Polym. Chem., 40, 1868 (2002).

19. J. Chen, Y. Maekawa, M. Yoshida, and N. Tsubokawa, Polym. J., 34, 30 (2002).

20. J. Chen, G. Wei, Y. Maekawa, M. Yoshida, and N. Tsubokawa, Polymer, 44, 3201 (2003).
21. D. Rivin, Rubber Chem. Technol., 36, 729 (1963).

22. J. Chen and N. Tsubokawa, Polym. Adv. Technol., 11, 101 (2000).

23. M. Okazaki, K. Maruyama, M. Tsuchida, and N. Tsubokawa, Polym. J., 31, 672 (1999).

24. J. Chen and N. Tsubokawa, Polym. J., 32, 729 (2000).

25. J. Chen and N. Tsubokawa, J. Appl. Polym. Sci., 77, 2437 (2000).

26. J. Chen and N. Tsubokawa, J. Macromol. Sci., Part A: Pure Appl. Chem., 38, 383 (2001).

27. N. Tsubokawa, A. Yamada, and Y. Sone, Polym. Bull., 10, 62 (1983).

28. N. Tsubokawa, A. Yamada, and Y. Sone, Polym. J., 16, 333 (1984).

29. N. Tsubokawa, A. Yamada, and Y. Sone, Kobunshi Ronbunshu, 42, 509 (1985). 